\title{
Fortalecimiento de la competitividad Institucional a través de la calidad en la educación: Caso Universidad del Zulia
}

\author{
Clemenza, Caterina* \\ Ferrer, Juliana** \\ Araujo, Rubén*** \\ Espina, Sabrina ${ }^{\star \star \star \star}$
}

\section{Resumen}

El objetivo general del presente trabajo es analizar la calidad de la educación universitaria como fundamento para fortalecer la competitividad institucional. Se presenta una revisión documental-descriptiva. Se abordan tres universos constituidos por los investigadores activos, los estudiantes de las distintas facultades y el sector externo que mantiene relación con las Unidades de Investigación de la Universidad del Zulia (LUZ). La muestra se seleccionó no probabilísticamente, para el caso de los investigadores y estudiantes; y a través de un censo para el sector externo. Se procedió a aplicar un instrumento estructurado a cada universo, contentivo de la variable estudiada. Los resultados indican que LUZ, concentra científicos y especialistas altamente calificados; cuenta con unidades de investigación donde se generan importantes programas y proyectos demandados por el sector productivo. En cuanto a la opinión de los estudiantes, se tiene que para ellos, las asignaturas potencian los valores éticos-morales y les desarrolla habilidades y destrezas; los profesores dominan y transmiten adecuadamente los contenidos, actualizan sus conocimientos y en ocasiones emplean material didáctico; mientras que la infraestructura presenta debilidades, obstaculizando el desempeño académico. Se concluye que, se hace necesario orientar acciones en la búsqueda de la calidad en las funciones básicas universitarias, integrando todos los actores de la institución y su interacción con el entorno; contribuyendo así, a insertar exitosamente nuestra universidad en un ambiente cada vez más competitivo que exige mejoramiento continuo, eficiencia, eficacia y efectividad social.

Palabras clave: Educación superior, calidad educativa, competitividad institucional.

\section{Recibido: 06-02-01. Aceptado: 06-09-20}

* Doctora en Ciencias Gerenciales. Profesora-investigadora Titular adscrita al Instituto de Investigaciones de la Facultad de Ciencias Económicas y Sociales de la Universidad del Zulia. Telf. 02617596513. E-mail: caterinaclemenza@yahoo.es. Autor para la correspondencia.

** Doctora en Ciencias Gerenciales. Profesora-investigadora Titular adscrita al Instituto de Investigaciones de la Facultad de Ciencias Económicas y Sociales de la Universidad del Zulia. Telf. 02617596513. E-mail: jumferrer@cantv.net

*** Magíster en Gerencia de Empresas, Economista, Comunicador Social. Asistente de Investigación. E-mail: raraujove@yahoo.es

**** Magíster en Gerencia de Empresas. Lic. en Administración. Asistente de investigación. 


\section{Strengthening Institutional Competitiveness Through Quality Education: The Case of the University of Zulia}

\section{Abstract}

The general objective of this paper is to analyze the quality of university education as a basis for strengthening institutional competition. It is a descriptive-documentary study. Three populations are studied, made up of active researchers, students from the different faculties, and the external sector that maintains contact with research units within the university (LUZ). The sample selected was nonprobabilistic, in the case of professors and students, and a census for the external sector. A structured instrument was applied to each population covering the variables under study. The results indicate that LUZ has groups of highly qualified scientists and specialists who belong to research units that generate important programs and projects requested by the productive sector. . In relation to the opinion of students, they indicated that their courses promoted ethical and moral values and developed abilities and skills; that professors understand and transmit adequately course contents, are up to date and occasionally apply didactic material; however that university infrastructure was weak, prohibiting academic development. The conclusion is that it is necessary to orient action towards maintaining quality in basic university functions, integrating all the actors in the institution and their interaction with the external ambient, contributing in this manner to successfully inserting the university in an environment which is more and more competitive and which requires continuous efficiency, efficacy and social effectiveness.

Key words: Higher education, educational quality, institutional competitiveness.

\section{Introducción}

El término calidad de la educación se asocia a la capacidad, tanto de las instituciones educativas como de los individuos que la conforman, de satisfacer los requerimientos del desarrollo económico, político y social de la comunidad en la que interactúa.

Particularmente, la Constitución Nacional establece en los artículos 102 , 103 y 106, además del derecho a la educación, la exigencia sobre ésta en cuanto a integralidad, calidad y pertinencia en igualdad de condiciones y oportunidades (Vázquez, Ferrer y Clemenza, 2001).

Para Tunnermann (1998), la calidad educativa es un concepto que requiere ser desagregado para poder analizar sus componentes y luego actuar sobre los mismos: el currículo, los métodos de enseñanza, los medios, la formación de los profesores, el ambiente pedagógico, la investigación educativa.

Puede decirse que la calidad de la educación es un concepto dinámico, cambiante en el tiempo; es diverso, pues varia según el contexto social, entre países y dentro de éstos, es multidimensional, puesto que es producto de diversas condiciones y es total, al atender las diferentes dimensiones del aprendizaje, como son la cognoscitiva, la socioafectiva, la psicomotora y los procesos intervinientes para lograrlos (Arríen, 1997).

Se puede indicar que la calidad es un término ambiguo el cual depende de la percepción del sujeto involucrado en el 
proceso, o sea, el cliente. Por lo tanto, cualquier definición que se intentase debe contar previamente con la identificación plena del cliente, o clientes, de las instituciones universitarias, lo cual se convierte en una tarea controvertida.

En el contexto de la educación superior, la utilización del concepto de calidad, según los requerimientos del cliente, genera varias interrogantes: "¿quién es el cliente de la educación superior? (la comunidad universitaria y/o el sector externo); ¿los estudiantes o las agencias que aportan recursos?; ¿los empleadores o los padres que pagan por la educación de sus hijos?; ¿y qué son los alumnos?; ¿clientes, productos o ambos? O quizás se debería hablar de los estudiantes como "consumidorores" de la educación, ya que son ellos quienes ingresan al sistema, "sufren" el proceso y emergen "educados". La otra pregunta que surge es, en caso de ser los estudiantes los clientes, ¿están ellos en posición de especificar los requerimientos del servicio? ¿Cómo son determinados esos requerimientos?" (González y Ayarza, 1997).

Se ha indicado reiteradamente que los usuarios por excelencia, y por lo tanto los clientes, son los propios estudiantes, otros consideran que un cliente importante es la propia comunidad universitaria. Adicionalmente, es indudable que son clientes tanto las instituciones públicas como las privadas. Y en forma global, no se duda que es la sociedad en su conjunto un cliente, particularmente importante, de las universidades. Cada uno de estos actores, estudiantes, empresas públicas y privadas, la comunidad universitaria y la sociedad en su conjunto, plantean diferentes requerimientos a la institución, re- querimientos a los que ésta debe darles respuesta (Tribus, 1993).

Una posición aceptada en esta materia, es la de considerar al estudiante un usuario, mas que un cliente, en virtud de que si bien recibe la acción universitaria, su función es la de transformarse con una ayuda invaluable de su parte, y constituirse en un resultado o producto del proceso de enseñanza aprendizaje universitario. Esta posición sostiene que los clientes son, la misma universidad, la sociedad, el gobierno, las empresas privadas y las empresas públicas (López, 1998).

Si bien el cliente externo es fundamental para cualquier institución, incluyendo las universidades, también es necesario considerar el cliente interno. A este respecto es importante, como dice Villarrroel (1997), garantizar la pertinencia interna. Esta se apoya en tres aspectos: liderazgo, estrategia académica y clima organizacional. El liderazgo, el cual no debe circunscribirse a las autoridades y dirigentes, permite dirigir el esfuerzo transformador y el trabajo en equipo. La estrategia académica debe procurar el ideal académico, que puede resumirse en las siguientes palabras "la capacidad de suspender en cada instante las propias valoraciones en beneficio del conocimiento científico; el poder prescindir de la propia posición, de la propia voluntad actual, en beneficio del conocimiento científico.

Al tratar de definir calidad en la educación superior, ésta se asocia con la reputación institucional que se tiene, a la disposición de los recursos académicos o financieros adecuados, a los resultados obtenidos, al valor intrínseco de los contenidos académicos, o por la apreciación 
del valor agregado que la institución brinda. En otras ocasiones, se identifica la calidad con la mayor o menor satisfacción por parte de una institución o programa, de los estándares fijados por las asociaciones profesionales, por las agencias de acreditación, o de manera más simple por la satisfacción manifiesta de los empleadores (Arríen, 1997).

La calidad en la educación superior, es asumida como un concepto multidimensional, que implica, además de la integración de todos los actores de la institución, involucrar los factores que conforman el sistema educativo y su interacción con el entorno social, económico, cultural y político. Así, la enseñanza, la investigación, el personal, los programas de aprendizaje y la infraestructura son elementos que deben ser considerados al elaborar programas, políticas y estrategias dirigidas a elevar la calidad de estas instituciones (Vázquez, Ferrer y Clemenza, 2001).

Sin embargo, en la concepción de que las universidades están obligadas a mejorar continuamente la calidad de los servicios que presta a la sociedad en el ámbito de sus funciones básicas: docencia, investigación y extensión, se incorporan aspectos, que de acuerdo a Arrarte (2003) afectan significativamente la búsqueda de la calidad en todos sus procesos; entre ellos:

- Diferenciación institucional. Existen Universidades públicas y privadas con regímenes legales diferenciados.

- Masificación de la matrícula. La masificación genera un proceso de superpoblación estudiantil que trae como consecuencia el deterioro de la calidad de la enseñanza.
- Restricción de Recursos Presupuestarios. Conlleva a una recurrente insuficiencia de recursos económicos que limita de manera significativa las iniciativas de desarrollo institucional.

- Incremento de la competencia entre instituciones de la Educación Superior. En el país existen diversidad de universidades pública, experimentales, privadas; así como también otros institutos de educación superior.

- Diversificación de la demanda. Ya no sólo los jóvenes recién egresados de la enseñanza secundaria aspiran a recibir una educación de nivel superior. Ahora también están los profesionales quienes buscan ampliar 0 mejorar sus conocimientos y destrezas, personas que desean o deben cambiar de ocupación, funcionarios y trabajadores que buscan acreditar y certificar su experiencia laboral y organizaciones interesadas en obtener servicios docentes en función de sus propios programas de capacitación.

- Transición hacia economías basadas en el conocimiento. El mayor de los cambios se ha producido en el campo del conocimiento y el mayor desafío es el de la educación ya que en el futuro inmediato se requerirá de personas diferentes, educadas de distinta manera como se hace hoy en día, con capacidades adecuadas para enfrentar y resolver situaciones nuevas en un entorno rápidamente cambiante.

- La búsqueda permanente de nuevos conocimientos y de nuevas técnicas dentro de una concepción unificadora del mundo, plantea la necesidad de revisar los esquemas tradicionales de la educación superior, su misión, su 
metodología, y en general, toda la estructura del sistema educativo.

- El cambio en la orientación de la valoración social. Nuestra sociedad ha experimentado cambios en sus valores: la calidad de los productos, la competencia, la responsabilidad pública, la transparencia y la eficiencia. El usuario asume en propiedad su rol de "cliente" y exige bienes y servicios de calidad, incluyendo una educación que satisfaga sus expectativas.

- Existe una creciente presión social sobre la calidad de los servicios universitarios: docencia, investigación, transferencia tecnológica, entre otras. Los cambios y transformaciones del entorno de la educación superior repercuten de manera directa en los sistemas de gestión, dirección y organización.

- Globalización de los mercados y competitividad. La creciente internacionalización de los mercados laborales en el ámbito profesional y técnico, exige que los diplomas otorgados en un país puedan ser reconocidos en los demás, para así facilitar la movilidad de la propia fuerza laboral más altamente calificada y atraer a estudiantes del extranjero por lo cual la evaluación de la calidad de las instituciones y programas de Educación Superior se ha transformado en un imperativo de alcance mundial.

La calidad de la educación universitaria puede explicarse, de acuerdo a Fernández (2002) como: un modelo de producción industrial que asume la educación como un proceso que puede igualarse al de producción industrial. Así, la calidad universitaria sería semejante a la calidad de los profesionales producidos para satisfacer las necesidades del sector externo. $Y$ un modelo de desarrollo global donde la calidad universitaria estaría determinada por el valor agregado que se generaría en el personal docente y administrativo, estudiantes, y en los servicios ofrecidos.

Puede observarse, como históricamente la calidad universitaria se ha venido valorando por parámetros muy concretos y cuantitativos, que pueden ir desde el número de estudiantes por aula, número de estudiantes por profesor, hasta las asignaciones presupuestarias, pero al pretenderse valorar los aspectos relacionados con los resultados, como puede ser el valor educativo agregado, o la adecuación de los estudiantes a las expectativas de trabajo, la misión se vuelve casi imposible, pues no se cuenta con los medios para determinar semejantes valores (Fernández, 2002).

La calidad de las universidades es el referente más importante para juzgar el desarrollo de estas instituciones. En la universidad venezolana no existen ni la normativa ni los mecanismos técnicos que permitan enjuiciar sistemáticamente la calidad de nuestras instituciones universitarias, lo que limita a nuestras universidades en los procesos de internacionalización y globalización (Proyecto SEA, 2002).

\section{Aspectos metodológicos}

En el trabajo se discute sobre la definición de calidad y competitividad universitaria; se presentan las potencialidades de la Universidad del Zulia para lograr ser competitiva; se determina la percepción del personal docente y de investigación en cuanto a los aspectos necesa- 
rios para que la Universidad del Zulia sea competitiva; se establecen las fortalezas, debilidades, amenazas y oportunidades de la Universidad del Zulia para lograr ser competitiva; se presenta la opinión de los estudiantes en cuanto a la calidad de los servicios prestados por la Institución; y la opinión del sector externo en cuanto a la calidad de los servicios prestados por las Unidades de Investigación de la Universidad del Zulia.

En la investigación se está en presencia de tres universos determinados por:

- El personal docente y de investigación que desarrollan sus labores en las diez Facultades y dos Núcleos de la Universidad del Zulia.

- Los estudiantes del último semestre o año de las Facultades de Agronomía, Arquitectura, Ciencias Económicas y Sociales, Ciencias Jurídicas y Políticas, Ciencias Veterinarias, Experimental de Ciencias, Ingeniería, Medicina, Odontología y Humanidades y Educación, durante el segundo semestre del año 2004.

- Las organizaciones del sector privado y público, que de acuerdo al Portafolio Extensionista de la Universidad del Zulia (2003), mantienen relación con las Unidades de Investigación de esta Institución.

Para la primera población, se trabajó con los investigadores que tienen proyectos financiados 0 registrados en el Consejo de Desarrollo Científico y Humanístico (CONDES), hasta febrero de 2005; los cuales ascienden a 1.280 investigadores. Se empleó una muestra no probabilística. Sin embargo, se deseó precisar el tamaño de la muestra para tener un parámetro de referencia de cuantos debía ser los investigadores a entrevistar, obteniéndose 97 sujetos. Luego, se utilizó un muestro por cuotas, donde se fijó un número determinado de investigadores a entrevistar por Facultad, resultando aproximadamente 8 investigadores de cada una de las 10 Facultades y los 2 Núcleos de la Universidad del Zulia (LUZ).

Para la segunda población, se empleó un muestreo no probabilística incidental, seleccionando directa e intencionalmente a 20 estudiantes entre 10 Facultades de LUZ, ascendiendo a un número de 200 estudiantes en total. La tercera población estuvo constituida por 27 organizaciones, que están relacionadas con la Unidades de Investigación de LUZ, para lo cual se realizó un censo de las mismas.

Para la recolección de información primaria se diseñaron tres instrumentos tipo entrevista, dirigido a cada una de la muestra seleccionada. Se seleccionó este tipo de instrumento ya que por la naturaleza de la investigación es el más apropiado, debido que el entrevistador puede verificar que el sujeto que responde el instrumento es la unidad de observación seleccionada.

El primer instrumento, dirigido a los investigadores, consta en la primera parte de 23 aseveraciones, con alternativas de respuestas: muy importante, importante, medianamente importante, poco importante, nada importante. La segunda parte, de cuatro preguntas abiertas. En el segundo instrumento, los estudiantes debían emitir su opinión respondiendo a 34 aseveraciones que se les presentaba. Por último, el tercer instrumento dirigido a los responsables de las organizaciones que mantienen relación con las unidades de investigación, constan de 9 preguntas, con alternativas fijas de respuestas. Para validar los instru- 
mentos se seleccionaron un grupo de 5 expertos, a fin de que determinaran si los reactivos estaban relacionados con los objetivos propuestos y si estaban bien redactas, entre otras observaciones. Además de la validez otro elemento a considerar al momento de aplicar un instrumento de medición es la confiabilidad.

Para efectos de este trabajo, el procedimiento aplicado para determinar la confiabilidad de los instrumentos se denomina confiabilidad por test-retest, la cual consiste en que un mismo instrumento de medición se aplica dos o más veces a un mismo grupo de personas, después de cierto período. Los tres instrumentos arrojaron una confiabilidad promedio del $82 \%$, indicando que los instrumentos utilizados son confiables para obtener la información que requiere este estudio.

\section{La Calidad: factor competitivo de la educación universitaria}

Debido a los grandes y acelerados cambios en todos los órdenes de la sociedad y a la desconfiguración de las fronteras, dadas por los procesos de globalización e internacionalización, las organizaciones se han visto en la urgente necesidad de incrementar su competitividad, para la cual, sus acciones apuntan a la búsqueda de estándares óptimos de operatividad que le permitan la interrelación transparente tanto con sus clientes internos como externos.

La gestión de la calidad proporciona principios que permiten lograr una continua evaluación de los estándares alcanzados con el fin de garantizar esa competi- tividad. Esta evaluación permite ofrecer productos y servicios con alto contenido valorativo; incorporar a los clientes, proveedores y competidores como agentes vitales dentro de la organización.

Se entiende por competitividad en el subsistema de educación universitaria, el nivel en que ésta crea valor, a través de una eficaz gestión de sus recursos y capacidades La calidad como factor de competitividad debe ser analizado en el contexto de los procesos sociales y políticos en los que interactúan agentes y actores.

A tal efecto, y de acuerdo a las entrevistas realizadas, para que la Universidad sea competitiva se requieren aspectos tales como:

- Dar a conocer a los usuarios de los servicios de la Universidad la seguridad de que existen unos niveles mínimos de calidad.

- Adecuación de la infraestructura a los requerimientos de la demanda.

- Desarrollar programas de enseñanza-aprendizaje que califiquen a los estudiantes para las exigencias del mercado laboral; asimilen conocimientos y participen en los procesos de cambio.

- Desarrollar la investigación básica y aplicada con pertinencia social.

- Capacidad para aprovechar, desarrollar, adaptar y utilizar los conocimientos derivados de las nuevas tecnologías.

- Formación del personal docente y de investigadores como elemento indispensable para lograr una política viable dentro de la gerencia de la educación superior que motorice los procesos.

- Contar con políticas eficientes de financiamiento. 
- Contribuir con las estrategias locales, regionales y nacionales de desarrollo regional.

- Diversificar su oferta educativa a fin de dar respuesta a las necesidades del mundo del trabajo.

- Fortalecer las relaciones de cooperación internacional que impulsen los convenios que sirvan de soporte financiero para las universidades

- Fortalecer la cooperación internacional con los otros centros de investigación de educación superior y con las oficinas de cooperación internacional de los gobiernos nacionales e internacionales.

Para Pérez (2004), la Universidad puede ser más competitiva si apunta sus estrategias a:

- Preparar a la comunidad universitaria para asimilar conocimientos y participar en un proceso de cambio permanente y rápido.

- Mejorar el capital humano

- Mejorar el capital tecnológico

- Mejorar la inserción laboral de sus egresados a través de una formación adecuada para que contribuyan a la mejora del sector productivo.

- Reforzar la investigación con pertinencia social.

- Reforzar la relación UniversidadSector productivo.

- Contribuir a las estrategias locales y regionales para el desarrollo de la sociedad del conocimiento.

Por ello al hablar de calidad para competir se puede hacer desde dos puntos de vista: una intrínseca, que hace referencia a la observación de la ciencia, y otra extrínseca, que se refiere al contexto social de desarrollo; que de acuerdo a Fernández (2002) se refiere a la pertinencia, es decir, a la correlación con las necesidades del entorno. Contemplar las dos visiones al mismo tiempo, supone observar un panorama multidimensional de la calidad y comprender que cada Universidad puede tener unos fines y demandas propios, matizando sus propios requerimientos de mejora de la calidad en base a las siguientes dimensiones:

- Dimensión académica; tiene como meta la excelencia a partir de estándares que fijan cada una de las áreas o disciplinas. Unida a esta dimensión iría la acreditación como forma de asegurar que se cumplen unos de los estándares previos mínimos de calidad.

- Dimensión de la reputación; se refiere a la imagen de calidad que transmite la Universidad a la sociedad en general.

- Dimensión de la perfección; implica el estudio y la optimización de los recursos y capacidades con los que cuenta la Universidad.

- Dimensión económica; estará relacionada con: La calidad como disponibilidad de recursos, tanto la accesibilidad a recursos existentes, como capacidad de captar nuevos. Y la calidad como consecución de objetivos. Para ello, habrá que poner énfasis en la calidad de los productos y servicios que ofrece la Universidad, así como en el uso óptimo de los recursos y capacidades.

- Dimensión de satisfacción de usuarios; supone llegar a cubrir los requerimientos y expectativas de los clientes de la Universidad, tanto actuales como futuros. Esta dimensión toma 
en cuenta especialmente a los estudiantes ya que de acuerdo a sus intereses y necesidades se deben tomar decisiones sobre las prioridades y evaluación de la calidad. Tomando en consideración la relevancia profesional; es decir, alcanzar los requisitos y expectativas del campo profesional para el cual los alumnos están siendo preparados y flexibilidad, es decir responder con rapidez a requerimientos de los clientes y nuevos avances dentro del campo profesional (Fernández, 2002).

- Dimensión organizacional; implica nuevas formas de gestión en la Universidad, que sean más emprendedoras y que apunten a una verdadera transformación institucional adecuada a los nuevos escenarios sociales. Se asocia con la necesaria habilidad de la institución para desarrollar e implementar normativas que satisfagan los requerimientos de la calidad educativa deseada.

Así, la autonomía y la diversificación universitaria obligan a las universidades a definir su propia estrategia y sus propios objetivos a fin de lograr ser competitivos. La calidad, en este caso, se mide por la capacidad de los equipos de gobierno en conseguir esas metas que ellos mismos han diseñado.

\section{Resultados de la información de campo}

\subsection{Potencialidades de la Universidad del Zulia para lograr ser competitiva}

La Universidad para ser competitiva debe apuntar a generar y expandir capacidades endógenas para sostener su desarrollo, inserta en una creciente globalización e internacionalización. La Universidad del Zulia no escapa a esta perspectiva, de allí que se hace necesario construir y perfeccionar sus capacidades, así como desarrollar sus potencialidades; a fin de lograr competir local, nacional e internacionalmente. Para ello, debe evaluar los propósitos de la política de investigación vigente, y determinar realmente, cuales son las potencialidades que posee, en cuanto a talento humano, infraestructura, calidad de la docencia e investigación, redes de investigación a las cuales se inserta, fuentes de financiamiento para desarrollar sus actividades de docencia, investigación y extensión, entre otras.

Hasta el mes de mayo de 2005, en la Universidad del Zulia (LUZ), se cuenta con 180 unidades de investigación. En estas unidades de investigación se ejecutan actividades derivadas de las investigaciones como: prestación y asistencia técnica, que se ofrece sobre todo en el manejo de la tecnología; desarrollo de prototipos; cursos de entrenamiento destinados al desarrollo profesional del recurso humano y al conocimiento de nuevas técnicas y enfoques que se deben aprender para la mejora continua.

Por otra parte, el CONDES ha registrado y financiado programas y proyectos que han impulsado la investigación científica en LUZ, y que de alguna manera han permitido la presencia de la universidad a nivel nacional e internacional a través de la relación con diversos organismos e instituciones que manifiestan un marcado interés por las actividades en este sentido desarrolladas por 
esta casa de estudios superiores. En la Tabla 1, se observa en detalle los programas y proyectos aprobados y los activos. Una parte, del total de estos programas y proyectos, son realizados a través de convenios y cooperación nacional e internacional.

Entre los organismos nacionales que cooperan con la investigación científica en LUZ, se encuentran: Instituto de Operación y Catálisis, Colegio de Abogados, Química Venoco, Reacción de Adquisición de Venceno, Laboratorio de Resonancia Magnética Nuclear, Planta experimental de tratamiento del agua, FEGALAGO, Laboratorio de Química de Metales de Transición, IVIC, Instituto Na- cional de investigaciones Agroalimentarias, Empresa Foodtech de Venezuela, Sistema Regional de Salud, Centro Frutícula, PDVSA, PEQUIVEN, ENELVEN, Gobernación del Estado Zulia, Alcaldía de Maracaibo, CORPOZULIA, Asociación Nacional de Cooperativas, CONAVI, CORPOZULIA, GADEMA, Alcaldía de Machiques, CECOSESUL, FUSAGRI, ICLAM, IRDEZ, CAICOC, Laboratorios Fyser, Roche Sheering y Plough Glaxi, PLANIMARA, Asobúfalo, VIATECA, Alcaldía de Mara, Fundación Polar.

$Y$ entre los organismos internacionales se encuentran: Mercantil Internacional (UNCITRAL) (EE.UU.), Departamento de Química y Tecnología de Alimento (Es-

\section{Tabla 1}

Número de Programas y Proyectos aprobados por el CONDES por Facultad en LUZ

\begin{tabular}{lccccc}
\hline Facultades & \multicolumn{5}{c}{ Años } \\
\cline { 2 - 6 } & $\mathbf{2 0 0 0}$ & $\mathbf{2 0 0 1}$ & $\mathbf{2 0 0 2}$ & $\mathbf{2 0 0 3}^{*}$ & $\mathbf{2 0 0 4}$ \\
\hline Agronomía & 14 & 6 & 6 & 11 & 12 \\
Arquitectura & 4 & 10 & 4 & 5 & 2 \\
Ciencias Económicas y Sociales & 16 & 20 & 19 & 4 & 29 \\
Ciencias Jurídicas y Políticas & 22 & 20 & 20 & 14 & 29 \\
Ciencias Veterinarias & 11 & 2 & 5 & 2 & 25 \\
Experimental de Ciencias & 30 & 15 & 36 & 2 & 52 \\
Humanidades y Educación & 39 & 26 & 39 & 23 & 37 \\
Ingeniería & 14 & 9 & 7 & 3 & 33 \\
Medicina & 25 & - & 16 & 2 & 26 \\
Odontología & 6 & 19 & 3 & 3 & 5 \\
Núcleo Punto Fijo & 2 & 1 & 4 & 2 & 3 \\
Núcleo Cabimas & 5 & 2 & 2 & 5 & 6 \\
Total & 175 & 130 & 161 & 75 & 259 \\
Programas y Proyectos Activos & 334 & 328 & 444 & 393 & 681 \\
\hline Fuente: Adaptado de: CONDES-LUZ. Memoria y Cuenta. Años 2000-mayo 2005. Proyectos aprobados se refiere \\
a financiados y registrados. * No hubo Convocatoria. & & & &
\end{tabular}


paña), CEPIS (Perú), OHIO State University Departamento de de Medicina Veterinaria (EE.UU.), Red Científica Latinoamericana ASHI (EE.UU.). Universidad de California (EE.UU.), Universidad de Cádiz y Sevilla (España), Universidad Autónoma de Barcelona (España), Universidad Alcalá de Henares (España).

Tal y como se observa, las actividades de investigación en la Universidad del Zulia son apoyadas, no solo desde el punto de vista financiero, sino también a través de convenios de cooperación nacional e internacional, por diversas instituciones, quienes tienen un marcado interés por los productos generados en las Unidades de Investigación. Esta cooperación está representada básicamente en utilización y aplicación de nuevas tecnologías, intercambio de personal docente y de investigación, elaboración de tesis, asesorías, análisis de datos, inserción de investigadores y docentes extranjeros para dictar cátedras en las Facultades y desarrollar investigación en las Unidades, entre otras.

En cuanto al talento humano dedicado a la investigación, se tiene un total de 1280 investigadores encargados de desarrollar esta actividad. Al comparar esta cifra con la del total de profesores de LUZ, el cual asciende a 2916 profesores activos (CEDIA, 2004) ${ }^{1}$, clasificados en ordinarios, contratados y becarios, se puede afirmar que cerca del $44 \%$ son investigadores activos. Cifra significativa, que infiere que los profesores se están dedicando cada vez más a la investigación.
Se observa como se está tomando conciencia de la importancia de generar conocimiento por medio de la investigación científica, tratando de darle prioridad, en términos de pertinencia social y factibilidad de la investigación; observándose una posición diferente del profesor al entender que la investigación es el elemento esencial y la docencia resultaría desfasada del contexto si no se vincula con el desarrollo de la ciencia, donde la investigación científica incorporada a la docencia, permite transmitir conocimientos generados en su propio trabajo, haciendo esta actividad más dinámica y contextualizada.

Este grupo de investigadores posee una formación permanente y participan activamente en el quehacer académico, generando y difundiendo conocimiento y con alta productividad académica que les ha merecido reconocimiento, tanto internacional como nacional. Específicamente a nivel nacional, la Fundación para el Avance de la Ciencia y la Tecnología (FONACIT), a través del Sistema de Promoción al Investigador (PPI), reconoce y premia los esfuerzos de los investigadores venezolanos.

Otro indicador importante de señalar es la calidad de las publicaciones científicas de la Universidad, donde la Universidad del Zulia (LUZ) es reconocida a nivel nacional e internacional por la calidad de las publicaciones científicas que edita, esto se afirma al observar la inclusión de las mismas en las bases de datos e índices nacionales e internacionales mas importantes de Latinoamérica y Europa, lo 
cual les ha permitido posicionarse en un contexto tan exigente como el científico.

Otro aspecto que se debe presentar son los programas de postgrado desarrollados en la Universidad del Zulia (LUZ) y que de alguna manera se han convertido en puntales para lograr que LUZ, se inserte en los espacios globales. De acuerdos a datos suministrados por el Consejo Central de Postgrado (2004), LUZ cuenta con 136 programas de postgrado (Doctorados, Maestrías y Especialidades), en áreas de conocimiento como: Ciencias Básicas, Ciencias Sociales, Ciencias de la Educación, Ingeniería, Tecnología, Arquitectura; Ciencias del Agro y Ciencias de la Salud.

Una parte importante de estos programas de postgrado se encuentran actualmente acreditados. Este es un aspecto de suma importancia para los participantes de dichos programas, permitiéndoles a los estudiantes extranjeros convalidar sus estudios en el país de origen, y a los nuestros poder continuar estudios en el exterior. Así la acreditación de los postgrados se convierte en la piedra angular en la fase de internacionalización de LUZ.

Tal y como se ha descrito, en la Universidad del Zulia, se concentra un número importante de científicos y especialistas altamente calificados en diversas áreas del conocimiento quienes desarrollan investigación; se cuenta con unidades de investigación donde se desarrollan importantes programas y proyectos; existen programas de postgrados acreditados y se editan revistas científicas donde se difunden los avances que en materia de investigación y desarrollo se realizan en LUZ.

\subsection{Opinión del personal docente y de investigación de la Universidad del Zulia}

En esta sección se presentan algunos resultados donde se visualiza la percepción de algunos docentes-investigadores de la Universidad del Zulia, referido a los aspectos necesarios presentes en las universidades para enfrentarse a los requerimientos del nuevo contexto, que demanda la calidad como factor competitivo; destacando como los más importantes: redes de investigación a distancia; la vinculación con los sectores productivos; ofrecimiento de servicios correspondientes con el desarrollo regional; desarrollo de proyectos estratégicos que respondan a las necesidades del entorno; formar personal docente y de investigación que motoricen los procesos; formadora del talento humano en el nuevo contexto internacional; fortalecimiento de las relaciones de cooperación internacional que impulsen los convenios los cuales sirven de soporte financiero para las universidades y fortalecimiento de la cooperación internacional con los otros centros de investigación de educación superior y con las oficinas de cooperación internacional de los gobiernos nacionales e internacionales.

Al detallar los resultados arrojados por la entrevista realizada, se puede observar como los sujetos refieren que las redes de investigación a distancia son indispensables para garantizar equipos interdisciplinarios que coadyuven al éxito de cualquier iniciativa de promover la ejecución de proyectos conjuntos entre diferentes entes de investigación nacional e internacional. 
Al expresar la necesidad de vinculación con el sector productivo, manifestaron una alta preocupación por la débil capacidad de respuesta de nuestra universidad frente a las demandas en ciencia y tecnología y particularmente de tiempos oportunos en las asesorías y consultorías, dificultad que obstaculizan la relación universidad-empresa.

Respecto a la oferta de servicios/productos de calidad correspondientes con el desarrollo regional, y el desarrollo de proyectos estratégicos relacionados a las necesidades del entorno, los docentes-investigadores, refieren que nuestra universidad se encuentra muy lejos de crear mecanismos que permitan la pertinencia con el entorno, destacando que en ocasiones los proyectos y programas de investigación se realizan mas por inquietud de los investigadores que por exigencias de lineamientos de políticas en materia de investigación y desarrollo.

Refiriéndose a las relaciones de cooperación internacional que impulsen los convenios que sirvan de soporte financiero para las universidades y a la cooperación internacional con los otros centros de investigación de educación superior y con las oficinas de cooperación internacional de los gobiernos nacionales e internacionales; los entrevistados señalaron mantener y fortalecer estas relaciones, debido que hasta la fecha, los convenios formales establecidos son muy pocos y no satisfacen las demandas particulares de los miembros de la comunidad universitaria. De allí, se hace necesaria una política interinstitucional que garantice el apoyo académico y financiero para lograr que los convenios de cooperación respondan a las necesidades académi- cas, donde no solo hayan beneficios aislados, sino que todos los involucrados en el proceso educativo universitario tengan la oportunidad de participar de ellos.

Otro aspecto referido es el desarrollo del talento humano, definido a través de la formación permanente y actualización sobre nuevos procesos del conocimiento dirigidos a toda la comunidad universitaria, en especial al personal docente y de investigación. Es importante destacar la necesidad de formar un personal altamente calificado, capaz de satisfacer las necesidades de todos los sectores de la actividad humana, desarrollando programas de relevante calificación que combine conocimientos de alto nivel, competencias y destrezas ajustadas a las necesidades presentes y futuras.

En cuanto al desarrollo de proyectos estratégicos que responden a necesidades del entorno, y promotora de integración entre áreas del conocimiento desarrolladas en centros de investigación; la universidad se debe convertir en potenciadora de la región, configurando un perfil que responda a las exigencias de la sociedad del futuro.

Es decir, la universidad del nuevo contexto asegurará las posibilidades de crecimiento, desarrollo y consolidación de los espacios a través del impulso de la ciencia y la tecnología, formando así mismo individuos con una visión flexible ante los cambios no previstos. Esa universidad necesaria está llamada por ello a generar un pensamiento innovador y condiciones que sustenten la competitividad y calidad de vida de la sociedad.

En cuanto a las fortalezas, que consideran posee la Universidad para ser competitiva, argumentan las siguientes: 
- Prestigio Institucional.

- Calificación y capacitación permanente del talento humano.

- Equipos de investigación consolidados.

- Diversidad de la oferta de carreras acordes con las demandas del país.

- Existencia de un Parque Tecnológico Universitario.

- Universidad emprendedora que apunta a una verdadera transformación institucional adecuada a los nuevos escenarios sociales.

- Alta productividad y producción científica.

- Imagen de calidad que transmite a la comunidad en general.

Por otra parte señalan ciertas debi-

lidades entre las cuales se encuentran:

- Deficiencias en las políticas para determinar prioridades de investigación.

- Ausencia de una agenda de investigación acorde con las políticas públicas.

- Poca generación de productos con pertinencia social.

- Unidades de Investigación poco dotadas con equipos de última generación.

- No cubre las expectativas de los clientes de la Universidad.

- Deficiente política comunicacional intra y extra universitaria.

- Infraestructura física no acorde con las actividades que realiza.

- Poca formación de personal de relevo.

Indicaron que la universidad se enfrenta a amenazas que impide insertarse con éxito en el contexto; entre ellas:

- Falta de estrategia institucional.
- Resistencia al cambio.

- Presencia de universidades privadas y otros institutos de educación superior.

- Un escenario para las actividades universitarias cada vez de más competencia global y no local.

- Baja credibilidad y confianza por parte del sector productivo, hacia las respuestas oportunas del sector universitario respecto a solución de problemas concretos.

- Presencia de conflictos gremiales que paralizan las actividades

Entre las oportunidades destacan:

- Demanda de servicios por parte del sector externo.

- Tendencia a la firma de convenios interinstitucionales entre organismos nacionales e internacionales y universidades públicas y privadas.

- Reconocimiento por parte de CNUOPSU a la producción y productividad de la Universidad del Zulia.

Estos resultados invitan a reflexionar, sobre los grandes retos que enfrenta la universidad, enmarcada en los procesos de globalización, el crecimiento económico basado en el conocimiento y la revolución de la comunicación e información. Estos retos pueden ser percibidos como terribles amenazas u oportunidades, para los cuales hay que estar preparados y anticiparse a los hechos, con una visión de futuro y con programas de calidad, innovadores y flexibles, y con alto sentido de pertenencia donde la universidad asuma la responsabilidad que la sociedad en su conjunto le demanda. 


\subsection{Opinión de los estudiantes de pregrado en cuanto a la calidad del servicio educativo ofrecido por la Universidad del Zulia}

A continuación se indaga sobre la percepción de los estudiantes en cuanto a sí los servicios que la Universidad del Zulia les presta para desarrollar su potencial y egresar con un título académico, son de calidad. Para ello se presentan categorías como la actuación del personal docente, la calidad de los programas académicos de las asignaturas, los diferentes servicios estudiantiles de apoyo a la academia, el ambiente físico, la infraestructura tecnológica y la actuación del personal administrativo y obrero.

La percepción es un elemento muy complejo, donde entra en escena la valoración de cada individuo respecto a la situación presentada. De tal manera que los estudiantes expresarán su valoración de acuerdo a la percepción que tengan de la realidad en la cual se desenvuelven y de la interacción con los miembros de la comunidad universitaria.
En la Tabla 2, se observa, de acuerdo a la opinión de los estudiantes, que los profesores dominan los contenidos programáticos, actualizan sus conocimientos y transmiten adecuadamente los contenidos. Mientras que un grupo manifestó que en ocasiones usan material didáctico en el dictado de sus clases y nunca usan dinámicas grupales.

Para elevar la calidad de la educación superior se hace necesario, entre otros aspectos, contar con personal docente y de investigación preactivos y atentos a las señales de cambio del entorno, Como lo dice la UNESCO (1998), proporcionar información fluida e interactiva, capaz de generar una mente curiosa y creativa; inspirar la creatividad y desarrollar valores en el individuo; proveer al estudiante de conocimientos y habilidades necesarias para producir ciencias básicas y tecnología avanzada.

En la Tabla 3, se visualiza como los estudiantes refieren que las asignaturas dictadas, potencian los valores éticos-morales y permite el desarrollo de habilidades

\section{Tabla 2}

Actuación del personal docente de la Facultad

\begin{tabular}{|c|c|c|c|c|c|c|}
\hline \multirow[t]{2}{*}{ Categorías } & \multicolumn{2}{|c|}{ Siempre } & \multicolumn{2}{|c|}{ En ocasiones } & \multicolumn{2}{|c|}{ Nunca } \\
\hline & $\mathrm{Fr}$ & $\%$ & Fr & $\%$ & Fr & $\%$ \\
\hline Dominio de los contenidos & 192 & 96 & 8 & 4 & - & - \\
\hline Transmisión adecuada de los contenidos & 107 & 54 & 74 & 36 & 19 & 10 \\
\hline Conocimiento actualizado & 196 & 98 & 4 & 2 & - & - \\
\hline Uso de material didáctico & 84 & 42 & 105 & 53 & 11 & 5 \\
\hline Uso de dinámicas grupales & 6 & 3 & 96 & 48 & 98 & 49 \\
\hline Infiere los contenidos a la realidad & 92 & 46 & 68 & 34 & 40 & 20 \\
\hline $\begin{array}{l}\text { Despierta el interés hacia la investigación } \\
\text { científica }\end{array}$ & 64 & 32 & 57 & 29 & 79 & 40 \\
\hline
\end{tabular}

Fuente: Entrevista realizada. 


\section{Tabla 3 \\ Calidad de los programas académicos de las asignaturas desarrolladas en la Facultad}

\begin{tabular}{lccccccc}
\hline \multirow{2}{*}{ Categorías } & \multicolumn{3}{c}{ Siempre } & \multicolumn{3}{c}{ En ocasiones } & \multicolumn{2}{c}{ Nunca } \\
\cline { 2 - 8 } & Fr & $\%$ & Fr & $\%$ & Fr & $\%$ \\
\hline Permiten al estudiante enfrentarse a un entorno & 85 & 43 & 7 & 3 & 108 & 54 \\
cambiante y colmado de incertidumbre & & & & & & \\
Permite la reflexión y el pensamiento crítico & 96 & 48 & 59 & 30 & 45 & 22 \\
Permiten el desarrollo de habilidades y destrezas & 104 & 52 & 47 & 23 & 49 & 25 \\
Potencian los valores éticos-morales & 138 & 68 & 62 & 32 & - & - \\
Despierta el interés hacia la investigación científica & 79 & 40 & 98 & 49 & 23 & 11 \\
\hline
\end{tabular}

Fuente: Entrevista realizada.

y destrezas. Aseguran además, que estas asignaturas no les permiten enfrentarse a un entorno cambiante y colmado de incertidumbre, al igual que no les despierta el interés hacia la investigación científica.

Al respecto, se puede decir que los planes y programas de las unidades de enseñanza-aprendizaje, deben someterse a un proceso de revisión para modificarlos de manera que se adapten a las nuevas condiciones de la sociedad y del mercado global. La revisión debería incluir, además de los contenidos temáticos, las metodologías de enseñanza, la formación del profesorado, la investigación y la vinculación con el sector productivo. El propósito básico sería mejorar la calidad y hacerlos congruentes con los cambios que se están produciendo.

Para la UNESCO (1998), las universidades deben modernizarse tanto curricular como estructuralmente, adaptar el proceso de enseñanza-aprendizaje a las exigencias de la sociedad, asumir las nuevas concepciones de aprendizaje y apoyar el desarrollo de un sistema educativo que ofrezca formación permanente, bajo el de- sarrollo integral del individuo, en el sentido de cubrir aspectos como: conocimiento científico (aprender a conocer), destrezas profesionales (aprender a hacer), valores morales y humanos (aprender a ser) y el ejercicio de la responsabilidad ciudadana (aprender a convivir).

Por otra parte, el proceso enseñanza-aprendizaje, no se limita al intercambio de información académica, sino que incorpora la educación integral del individuo, donde todos los aspectos de su vida académica afectan significativamente su actuación. Entre estos aspectos, se encuentran los servicios de apoyo que permiten complementar su formación.

Al respecto, señalaron que en ocasiones los libros y documentos que solicitan en la biblioteca y hemeroteca se encuentran disponibles. Mientras que señalan que el horario de la misma para prestarle servicio, nunca se adapta a sus necesidades. Uno de los servicios mas importantes, es de la biblioteca, quien es considerada entre los elementos para evaluar la calidad de las instituciones de educación superior, donde se considera su capacidad, tanto por el volumen de 
textos, como por la actualización de los mismos; el espacio físico y la incorporación tecnológica.

Los servicios de reproducción, solo en ocasiones son los adecuados; los servicios de internet, fax, teléfonos nunca están disponibles y consideran que siempre hay disponibilidad de transporte (Tabla 4).

En otro orden de ideas, en la Tabla 5 , se observa la percepción de los estudiantes entrevistados en cuanto a la actuación del personal administrativo y obrero. Los estudiantes entrevistados, manifestaron que el lenguaje y la apariencia física del personal administrativo y obrero es siempre el más adecuado, además señalaron que demuestran respeto en el trato con los estudiantes y es receptivo y amable. Solo en ocasiones responden a las inquietudes de los estudiantes y están dispuestos a resolver los problemas que se les presentan.

El contacto directo que tienen los estudiantes con el personal administrativo y obrero, debe ser cuidado de manera particular, ya que éstos se convierten en el personal de apoyo de los estudiantes en todos los espacios universitarios. Esto es, a fin de que perciban de manera posi- tiva la calidad del servicio que se les brinda a la hora de solicitar cualquier servicio que en las diferentes Dependencias, Direcciones, Coordinaciones o Secretarias le puedan brindar.

En cuanto al ambiente físico, se puede señalar que se hace necesario contar con instalaciones apropiadas, agradables y seguras, donde la comunidad universitaria pueda desarrollar sus actividades cómodamente, para así obtener mayor rendimiento en sus labores. La Tabla 6 , muestra los resultados obtenidos, en la entrevista realizada, donde los estudiantes manifestaron que en ocasiones las instalaciones están en buenas condiciones al igual que los salones de clase; mientras que las condiciones físicas de la biblioteca no son las más adecuadas y hay inseguridad en el espacio físico.

En líneas generales, se observa en la Tabla 7 como la infraestructura tecnológica no es la apropiada para apoyar a los estudiantes en su quehacer cotidiano dentro de las Facultades, donde opinan que nunca cuentan con equipos audiovisuales adecuados para el dictado de las clases, equipos computarizados para prestrarles servicio, ni tienen acceso a

Tabla 4

Servicios estudiantiles prestados por la Facultad

\begin{tabular}{lcccccc}
\hline \multirow{2}{*}{ Categorías } & \multicolumn{3}{c}{ Siempre } & \multicolumn{2}{c}{ En ocasiones } & \multicolumn{2}{c}{ Nunca } \\
\cline { 2 - 7 } & Fr & $\%$ & Fr & $\%$ & Fr & $\%$ \\
\hline $\begin{array}{l}\text { Disponibilidad de textos y documentos en la biblioteca } \\
\text { y hemeroteca }\end{array}$ & 95 & 48 & 97 & 49 & 8 & 3 \\
Servicios de reproducción adecuados & & & & & & \\
Disponibilidad de servicios de teléfono, internet,fax & 46 & 46 & 98 & 49 & 10 & 5 \\
Horario de la biblioteca adecuado a sus necesidades & 32 & 16 & 63 & 32 & 105 & 52 \\
Disponibilidad de servicio de transporte & 104 & 52 & 94 & 47 & 2 & 1 \\
\hline
\end{tabular}

Fuente: Entrevista realizada. 


\section{Tabla 5}

\section{Actuación del personal administrativo y obrero de la Facultad}

\begin{tabular}{lccccccc}
\hline \multirow{2}{*}{ Categorías } & \multicolumn{3}{c}{ Siempre } & \multicolumn{3}{c}{ En ocasiones } & \multicolumn{2}{c}{ Nunca } \\
\cline { 2 - 7 } & Fr & $\%$ & Fr & $\%$ & Fr & $\%$ \\
\hline Lenguaje apropiado & 102 & 51 & 96 & 48 & 2 & 1 \\
Apariencia apropiada al trabajo que realiza & 134 & 67 & 66 & 33 & - & - \\
Responde a las inquietudes de los estudiantes & 87 & 43 & 98 & 49 & 15 & 8 \\
Es receptivo y amable & 104 & 52 & 82 & 41 & 22 & 7 \\
Brinda asesoría & 96 & 48 & 67 & 34 & 37 & 18 \\
Demuestra respeto & 107 & 54 & 90 & 44 & 3 & 2 \\
Dispuesto a resolver problemas & 84 & 42 & 92 & 46 & 24 & 12 \\
\hline
\end{tabular}

Fuente: Entrevista realizada.

Tabla 6

Ambiente Físico de la Facultad

\begin{tabular}{|c|c|c|c|c|c|c|}
\hline \multirow[t]{2}{*}{ Categorías } & \multicolumn{2}{|c|}{ Siempre } & \multicolumn{2}{|c|}{ En ocasiones } & \multicolumn{2}{|c|}{ Nunca } \\
\hline & $\mathrm{Fr}$ & $\%$ & $\mathrm{Fr}$ & $\%$ & Fr & $\%$ \\
\hline Instalaciones en buenas condiciones y agradables & 78 & 39 & 92 & 46 & 30 & 15 \\
\hline Espacio bien distribuido & 102 & 51 & - & - & 98 & 49 \\
\hline Salones de clase en óptimas condiciones & 85 & 43 & 99 & 49 & 16 & 8 \\
\hline Accesibilidad al estacionamiento & 106 & 53 & 94 & 47 & - & - \\
\hline Seguridad en el espacio físico & 28 & 14 & 35 & 17 & 137 & 69 \\
\hline Adecuadas condiciones físicas de la biblioteca & 89 & 44 & 15 & 8 & 96 & 48 \\
\hline
\end{tabular}

Fuente: Entrevista realizada.

Tabla 7

Infraestructura Tecnológica de la Facultad

\begin{tabular}{lccccccc}
\hline \multirow{2}{*}{ Categorías } & \multicolumn{2}{c}{ Siempre } & \multicolumn{2}{c}{ En ocasiones } & \multicolumn{2}{c}{ Nunca } \\
\cline { 2 - 6 } & Fr & $\%$ & Fr & $\%$ & Fr & $\%$ \\
\hline $\begin{array}{l}\text { Equipos audiovisuales adecuados para el } \\
\text { dictado de las clases }\end{array}$ & 38 & 19 & 29 & 15 & 133 & 66 \\
$\begin{array}{l}\text { Equipos computarizados para prestar ser- } \\
\text { vicio a los estudiantes }\end{array}$ & 8 & 4 & 15 & 8 & 177 & 88 \\
$\begin{array}{l}\text { Acceso del estudiante a bases de datos } \\
\text { relacionados con su actuación académica }\end{array}$ & - & - & - & - & 200 & 100 \\
$\begin{array}{l}\text { Laboratorios dotados de equipos de alta } \\
\text { tecnología }\end{array}$ & 98 & 49 & 76 & 38 & 26 & 13 \\
$\begin{array}{l}\text { Servicio de internet que le permita desa- } \\
\text { rrollar sus actividades investigativas }\end{array}$ & 101 & 51 & 9 & 5 & 90 & 44 \\
\hline
\end{tabular}

Fuente: Entrevista realizada. 
bases de datos relacionados con su actuación académica.

Así se observa que, para prestar un servicio de calidad, se debe considerar como el estudiante (usuario interno) percibe los aspectos tangibles e intangibles que le afectan directamente e impactan significativamente su desenvolvimiento académico. Así, los programas de aprendizaje, los docentes, el personal administrativo y obrero y la infraestructura física y tecnológica son elementos a tomar en cuenta, al elaborar programas, políticas y estrategias dirigidas a elevar la calidad de la educación en la Universidad del Zulia.

\subsection{Opinión del sector externo en cuanto a la calidad del servicio ofrecido por las Unidades de Investigación de la Universidad del Zulia}

Es importante dejar en claro que de las 27 organizaciones consideradas para este estudio como clientes externos, sólo se logró entrevistar a 17 organizaciones, por las siguientes razones:
- El personal encargado del contacto directo con las Unidades de Investigación se encontraba de viajes o suspendido.

- Mantienen confidencialidad con la relación que poseen con la Unidad de Investigación.

- Se negaron a colaborar con la investigación.

- Quien manejaba la información se encontraba en los departamento de la Oficina Principal en Caracas.

- Poco conocimiento en los empleados de las organizaciones sobre este tipo de actividades.

La Tabla 8 describe los beneficios que han recibido las organizaciones gracias a la relación que mantienen con las diferentes Unidades de Investigación de LUZ.

El sector externo considera que se beneficia con los servicios prestados por las Unidades de Investigación, cuando éstas les presta asesoría y asistencia técnica, le ofrece cursos de actualización, programas de capacitación, consultoría especializada, entre otras. Así las empresas se benefician directamente de los

Tabla 8

\section{Beneficios que recibe el sector externo por parte de las Unidades de Investigación de LUZ}

\begin{tabular}{lcc}
\hline Beneficios & Frecuencia & $\%$ \\
\hline Asesoría y asistencia técnica & 18 & 22 \\
Actualización del conocimiento científico-tecnológico & 14 & 18 \\
Posibilidad de asesoramiento gerencial & 3 & 4 \\
Posibilidad de asistencia multidisciplinaria & 6 & 7 \\
Entrenamiento del personal & 12 & 15 \\
Programas de capacitación & 14 & 18 \\
Consultoría especializada & 12 & 15 \\
Desarrollo de prototipos & 1 & 1 \\
\hline
\end{tabular}

Fuente: Entrevista realizada. 
servicios que la Universidad presta a través de su recurso humano y los servicios especializados que esta pueda ofrecer.

Las áreas demandadas vienen dada por los requerimientos de cada una de las empresas, quienes de acuerdo a su naturaleza, requieren servicios de particulares Unidades de Investigación. Así, las empresas requieren servicios de consultoría jurídica y a la vez necesitan que se les dicte curso a sus empleados relacionados con el manejo de una maquinaria, o se le haga a su empresa una evaluación del clima organizacional, entre otras.

En cuanto a la calidad del personal que labora en las Unidades de Investigación, los entrevistados afirmaron que éste es de calidad, argumentando que consideran que tiene capacidad técnica y están actualizados en los últimos conocimientos sobre avances científicos y tecnológicos.

En la Tabla 9 se hace referencia a la infraestructura física y tecnológica con las que cuentan las Unidades de Investigación, considerando la primera como el espacio físico donde se lleva a cabo la investigación y la segunda referida a la incorporación tecnológica en la labor investigativa.

Se observa como el $49 \%$ de la muestra manifiesta que la infraestructura que poseen las Unidades de investigación con las cual ellos se relacionan es poco adecuada, el 22\% indicó ser inadecuadas, solo el $29 \%$ opinó que eran adecuadas. La mayoría de los entrevistados señalaron que percibían un notable espacio reducido, deteriorado y mobiliario inadecuado.

En cuanto a la infraestructura tecnológica, se observa que la mayoría (88\%) afirma que ésta es adecuada para prestar un servicio de calidad, sin embar-

\section{Tabla 9}

\section{Infraestructura física y tecnológica de las Unidades de Investigación}

\begin{tabular}{lcc}
\hline Alternativas & $\begin{array}{c}\text { I. Física } \\
\%\end{array}$ & $\begin{array}{c}\text { I. Tecnológica } \\
\%\end{array}$ \\
\hline Adecuada & 29 & 88 \\
Poco adecuada & 49 & 12 \\
Inadecuada & 22 & \\
Totales & 100 & 100 \\
\hline
\end{tabular}

Fuente: Entrevista realizada.

go el resto manifestó que las Unidades de investigación deben estar dotadas de equipos que les permitan ser más eficaces en su labor (Tabla 10).

Se observa que la muestra del sector externo está de acuerdo en manifestar que entre las características de las Unidades de Investigación está su alta capacidad de respuesta a sus requerimientos, la atención personalizada que brindan y la satisfacción de sus necesidades. Solo consideran que los canales de comunicación no son los mas adecuados al momento de entablar la relación.

En otro orden de ideas se aprovechó la oportunidad para indagar sobre la opinión que les merecía los egresados de la Universidad del Zulia (LUZ). Al respecto, en las tablas siguientes se visualiza tal apreciación.

La muestra manifestó que ha contratado egresados de la Universidad del Zulia (LUZ) en diversas áreas, dependiendo de la actividad operativa requerida. Se observa, en la Tabla 11, como el área administrativa fue la más demandada seguida por ingeniería y contabilidad.

En cuanto al nivel académico de los egresados, se tiene que de las 17 empre- 


\section{Tabla 10 \\ Los servicios prestados por las Unidades de Investigación de LUZ se caracterizan}

\begin{tabular}{lccccc}
\hline Categorías & TA (\%) & MA (\%) & NAND (\%) & MD (\%) & TD (\%) \\
\hline Alta capacidad de respuesta & 59 & 41 & - & - & - \\
Atención personalizada & 82 & 18 & - & - & - \\
Acorde con las necesidades de los clientes & 100 & - & - & - & - \\
Adecuados canales de comunicación & 29 & 53 & - & 18 & - \\
\hline
\end{tabular}

Fuente: Entrevista realizada.

\section{Tabla 11 \\ Áreas que ha contratado egresados de LUZ}

\begin{tabular}{lcc}
\hline Alternativas & Fr & $\%$ \\
\hline Ingeniería & 10 & 28 \\
Administración & 12 & 33 \\
Economía & 3 & 8 \\
Jurídica & 2 & 6 \\
Contabilidad & 9 & 25 \\
\hline
\end{tabular}

Fuente: Entrevista realizada.

sas, el $83 \%$ considera que el nivel académico de los egresados de LUZ, que han tenido oportunidad de contratar, es excelente y muy bueno, el resto consideró que era bueno. Razón importante para mantener e incrementar los estándares de calidad de la enseñanza impartida, a fin de que este aspecto no se vea debilitado.

Al requerir la opinión de los entrevistados sobre la calidad académica de la Universidad del Zulia (LUZ), manifestaron estar totalmente de acuerdo que les permite desarrollar habilidades y destrezas necesarias para enfrentarse a un entorno de incertidumbre; así como se potencian los valores éticos-morales que les van a permitir conducirse y transformar organizaciones signadas por el flagelo de la corrupción. Sin embargo, consideran que se debe reorientar las carre- ras ofrecidas a fin de que estas respondan más a los intereses de los sectores productivos y del Estado (ver Tabla 12).

De acuerdo a lo expuesto surge la necesidad impostergable, de buscar la calidad en cada una de las funciones básicas universitaria, a fin de que la Universidad se situé en un lugar privilegiado y de referencia obligada en el contexto nacional e internacional. Para esto, se requiere de los esfuerzos de toda la comunidad universitaria para mejorar continuamente, y una actitud proactiva hacia el cambio.

\section{Consideraciones finales}

La Universidad, como institución en contacto permanente con su entorno, no escapa a las amenazas que le plantea el siglo XXI. El incesante cambio, la elevación de los niveles de competitividad, el aumento de la complejidad y la transformación en el rol que juegan los usuarios, la colocan en una situación comprometida, de tal manera que la sobrevivencia depende de la profundización de las acciones que deben acometerse para originar la transformación deseada, que adecue las estructuras de la Universidad, a los nuevos tiempos. En este sentido, evaluar la calidad se convierte en una arista importante para lograr la competitividad. 


\section{Tabla 12 \\ Calidad Académica de LUZ}

\begin{tabular}{|c|c|c|c|c|c|}
\hline Categorías & TA (\%) & MA (\%) & NAND (\%) & MD (\%) & TD (\%) \\
\hline $\begin{array}{l}\text { Las carreras ofrecidas por LUZ están acor- } \\
\text { des con las exigencias del mercado laboral }\end{array}$ & 18 & 82 & - & - & - \\
\hline $\begin{array}{l}\text { La educación recibida por los estudiantes } \\
\text { permite la reflexión y el pensamiento crítico } \\
\text { de los estudiantes }\end{array}$ & 30 & 70 & - & - & - \\
\hline $\begin{array}{l}\text { La educación recibida por los estudiantes } \\
\text { permite el desarrollo de habilidades y des- } \\
\text { trezas en los estudiantes }\end{array}$ & 88 & 12 & - & - & - \\
\hline $\begin{array}{l}\text { La educación recibida por los estudiantes } \\
\text { potencia los valores éticos-morales }\end{array}$ & 82 & 18 & - & - & - \\
\hline $\begin{array}{l}\text { La educación recibida por los estudiantes } \\
\text { permite al estudiante enfrentarse a un entor- } \\
\text { no cambiante y colmado de incertidumbre }\end{array}$ & 65 & 35 & - & - & - \\
\hline
\end{tabular}

Fuente: Entrevista realizada.

El aseguramiento de la calidad en las universidades, debe convertirse en una estrategia de gestión y mejora como forma de respuesta a la necesidad de estudios con pertinencia sobre la realidad circundante, que coadyuve a enfrentar las realidades socialmente construidas y promueva el cambio en los planes de estudio, en la investigación, en la formación del personal, y la infraestructura logrando una eficaz gestión de sus recursos y capacidades, que le permitan ser competitiva a nivel nacional e internacional.

Una universidad nueva, renovada, creativa; capaz de aceptar y asumir el reto de la transformación, liderizada internamente e irradiada hacia el exterior, tal y como el país lo exige y nuestra época también. Esa nueva universidad está urgida de reencontrarse a sí misma, bajo un claustro universitario que sobrepasó los espacios convencionales y académicos tradicionales, que necesita aprender a corregir, tener voluntad política para reformar, transformar y permitir avanzar a pesar de las crisis, en la búsqueda de una universidad al servicio de la investigación, donde el espacio para la formación de un profesional sea parte del compromiso social.

Por ello, el logro y la permanencia en el tiempo de la universidad, se consolidará, siempre y cuando, se establezca, instrumente y se desarrolle una sólida cultura organizacional, entendida como un sistema de valores internos a la institución, sustentada en la historia, en su misión creadora, crítica y transformadora, su visión, sus estrategias, conjuntamente con las creencias, los rituales, el estilo operativo y su clima político social; que apoye las estrategias planificadas para la conquista de los objetivos propuestos de carácter académico-administrativo, los cuales deben estar relacionados no solo docencia, investigación y extensión, sino también universidad y sociedad.

En cuanto a la formación de personal docente y de investigación y a la formación del talento humano en el nuevo contexto na- 
cional e internacional, se requerirán personas formadas de manera integral, con capacidades y habilidades técnicas, capaces de enfrentar y solucionar problemas en un entorno rápidamente cambiante, con pensamiento crítico y con sólidos valores morales. Al respecto, la Universidad del Zulia tiene que transformarse estructuralmente, se sugiere el cambio organizacional, principalmente en las carreras que se están ofreciendo, dado que hay exigencias del entorno social y productivo que demandan nuevos perfiles.

\section{Referencias Bibliográficas}

Albornoz Orlando (1997). "La cuestión de la productividad, rendimiento y competitividad académica del personal docente y de investigación en América Latina y el Caribe". En: La educación superior en el siglo XXI. Visión de América Latina y el Caribe, tomo I. Ediciones CRESALC/UNESCO. Caracas (Venezuela). Págs. 391-421.

Arrarte, Raúl (2003). La calidad y la productividad en la universidad estatal. www.bibliotecadigital UNMSM.pe.

Arríen Juan B. (1997). "Calidad y acreditación: exigencias alas universidades". En: La educación superior en el siglo XXI. Visión de América Latina y el Caribe, tomo I. Ediciones CRESALC/UNESCO. Caracas (Venezuela). Págs. 447-460.

Consejo de Desarrollo Científico y Humanístico (2003). La Oferta Extensionista de LUZ. La Universidad del Zulia. Maracaibo, Edo. Zulia.

Consejo de Desarrollo Científico y Humanístico (2000-2004). Memoria y Cuenta. La Universidad del Zulia. Maracaibo, Edo. Zulia.

Fernández R., Emilia (2002). "Cómo afrontar la mejoría de la calidad en la educación su- perior". Revista Esic Market. Número 111, Enero-Abril, España. Págs. 24-37.

González, Luis E. y Ayarza, Herman (1997). "Calidad, evaluación institucional y acreditación en la educación superior en la región Latinoamericana y del Caribe". En: La educación superior en el siglo XXI. Visión de América Latina y el Caribe, tomo I. Ediciones CRESALC/UNESCO. Caracas (Venezuela). Págs. 337-390.

López, Francisco (1998).Gestión de calidad en educación. Hacia unos centros educativos de calidad. En http: www. pnctic.mec.es.

Pérez, Francisco (2004). "La mejora de la competitividad: La contribución de las universidades". En: Memorias sobre la competitividad en la sociedad del conocimiento y las instituciones de ciencia y tecnología. Valencia, España.

Sistema de Evaluación y Acreditación de la Universidades Nacionales (SEA) (2002). Consejo Nacional de Universidades (CNU). Oficina de Planificación del Sector Universitario (OPSU). (Venezuela).

Tribus, Mirón (1993). Total Quality in Schools of Bisiness and of Engineering. Exergy, Inc. Hayward.

Tunnermann, Carlos (1998). La educación superior en el umbral del siglo XXI. Ediciones CRESALC-UNESCO. (Venezuela).

Vásquez, José; Ferrer, Juliana y Clemenza, Caterina (2001). "Transformación universitaria bajo la óptica de la Autoevaluación. Caso LUZ". En: Revista Ciencias del Gobierno. Año 5, No 10. Págs. 124-139.

Villarroel, César (1997). "Calidad y acreditación latinoamericana para Latinoamérica". En: La educación superior en el siglo XXI. Visión de América Latina y el Caribe, tomo I. Ediciones CRESALC/UNESCO. Caracas (Venezuela). Págs. 607-631. 